\title{
Sensitivity and accuracy in processing $x$-ray spectrum images
}

\section{P.J.Statham}

\author{
Oxford Instruments Analytical Limited, High Wycombe, Bucks HP12 3SE, U.K.
}

The ability to acquire a complete $\mathrm{x}$-ray spectrum at every image pixel provides opportunities for "data mining". Spectrum images are typically acquired from 128x128 up to 1024x1024 pixel resolution and if acquisition times are only a few minutes it is often possible for less than 100 counts to be recorded in a single pixel spectrum. Fig. 1 shows two low count $20 \mathrm{kV}$ spectra from the same material in the region where Fe and Ni peaks are expected. The few non-zero channels in each spectrum rarely coincide; as a consequence the cross correlation between spectra from two supposedly identical pixels can be close to zero, as it would be if the pixels were from different materials. Multivariate statistical techniques work on the basis of recognising systematic above random correlations and such very low count data make it difficult to resolve the non-noise eigenvalues from noise in an eigenanalysis approach.

Several other approaches to processing have been recently reviewed [1]. Although many are compromised by low count data. quantitative mapping in principle provides optimum performance provided rigorous background correction and peak profile fitting methods are used to determine elemental peak areas from each pixel. A further approach has been reported [2] where for each pixel, weighted sums from the spectrum give R,G and B values using three weighting curves as shown in fig. 2 and the result for each pixel is plotted on a ternary scatter diagram. This can reveal distinct phases where only overlapping L lines are available and total spectrum counts are below 100 counts per pixel [2]. The performance of this colour transform approach has been tested by simulating pixel spectra from two phases with different $\mathrm{Pb}$ and $\mathrm{S}$ content (Fig.3). Fig.4 shows representative single pixel spectra from the two phases. Fig.5 shows the results of a quantitative mapping approach providing $\mathrm{Pb}$ and $\mathrm{S}$ intensities for 100 pixels of each phase and Fig. 6 shows that despite the poor statistics and overlapping peaks, the colour transform approach still resolves the two phases as distinct clusters in the scatter plot.

Increased count rates are useful to overcome statistical limitations and will enable more techniques for phase discrimination [3]. For example, derived spectrum statistics at each energy channel, such as the Maximum over all pixels, can pick out small area features if statistics are good. The count rate can drop dramatically as the beam crosses trenches, gaps, voids, and regions in shadow and this can cause some electronic systems to give variable resolution and peak shift. Therefore, for accurate quantitation when peaks overlap it is important to work at count rates where spectral quality is maintained. At high count rates, with $\mathrm{Si}(\mathrm{Li})$ or SDD detectors, pile up can be significant [3] and the advantage of a quantitative mapping approach is that pile up correction can be applied to individual pixel spectra to remove sum peak artefacts[4].

\section{References}

[1] J.J.Friel and C.E.Lyman, Microsc. Microanal. 12, (2006) 2-25

[2] P.J.Statham, Microsc. Microanal 10 (Suppl 2), (2004), 1026CD

[3] D.E.Newbury.. Scanning, 27, (2005), 227-239

[4] P.J.Statham, Proc. EMAS/IUMAS 2005, Microchimica Acta, In press (2006) 


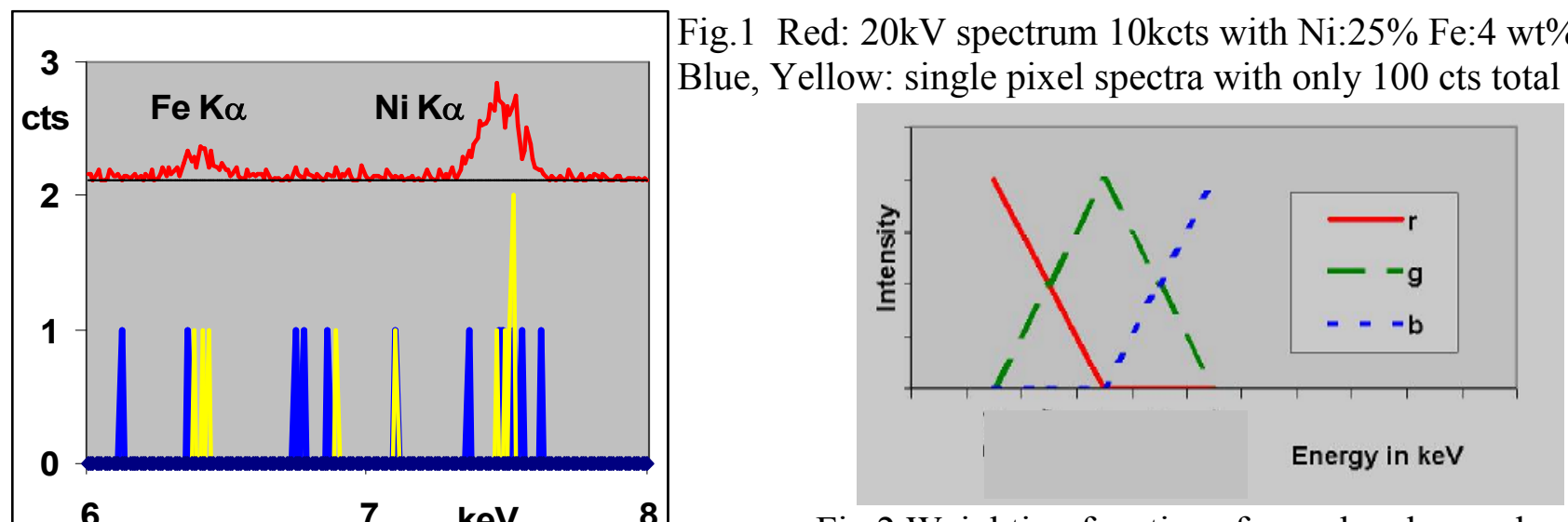

Fig.2 Weighting functions for r,g,b colour values
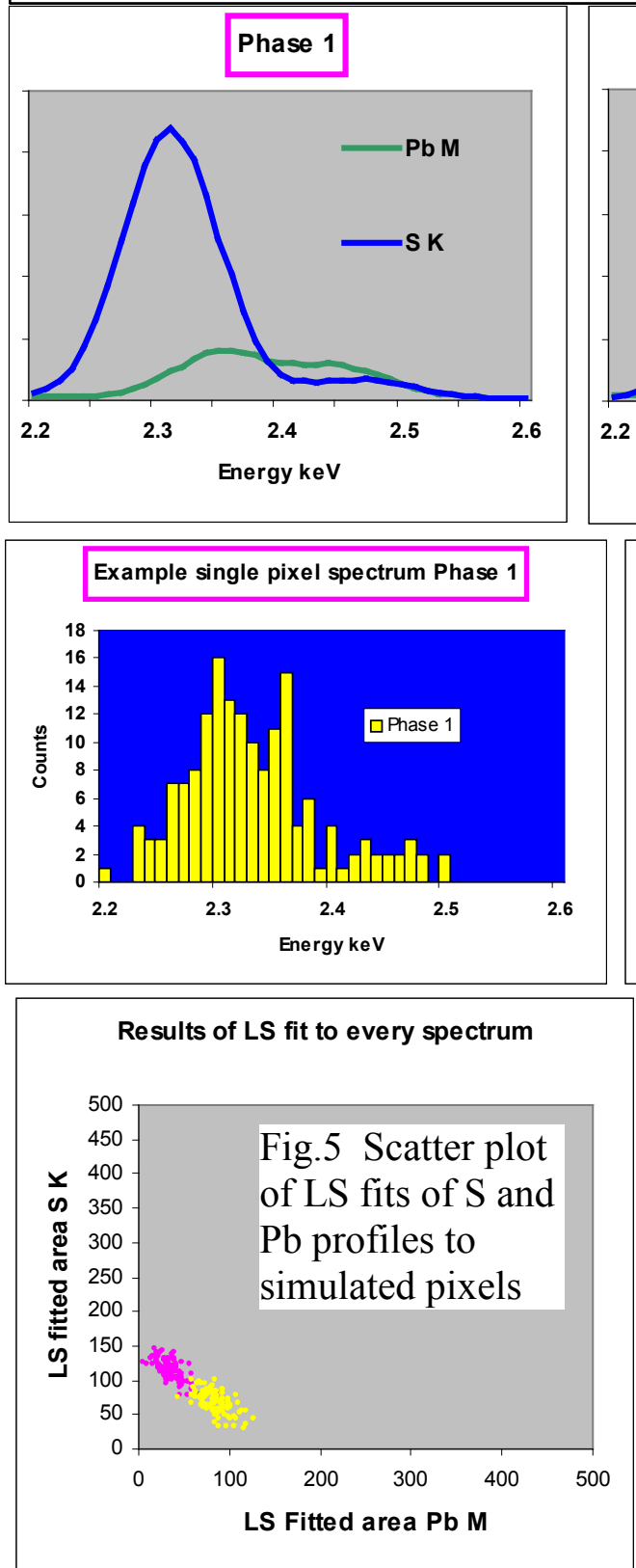
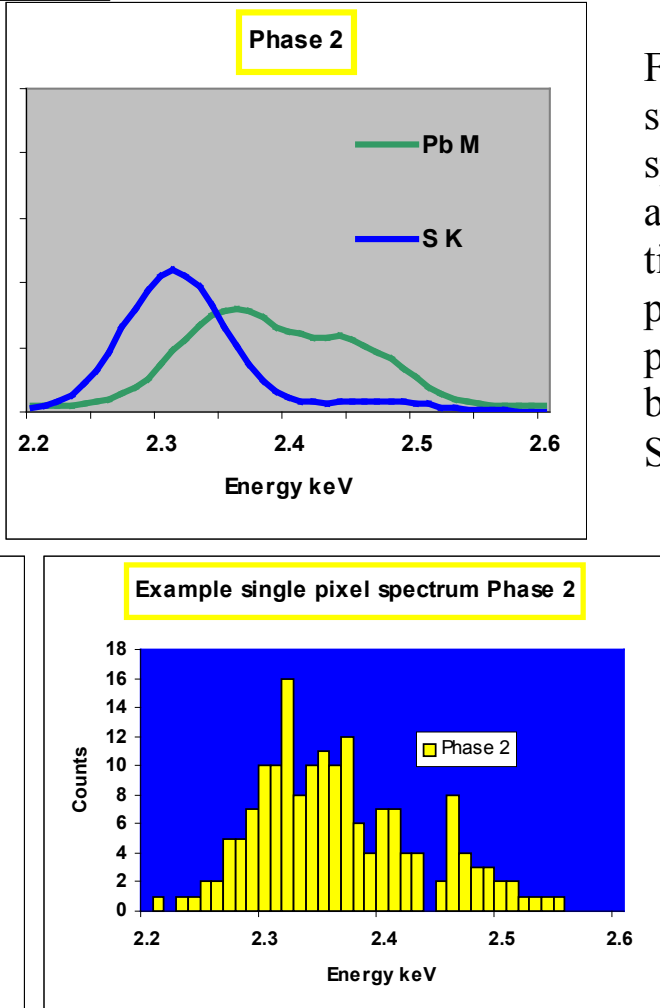

Fig.6 $\rightarrow$ Scatter plot of $\mathrm{R}, \mathrm{G}, \mathrm{B}$ values obtained as in fig. 2 for 100 pixels of each phase
Fig.3 Components summed to simulate spectra containing $\mathrm{Pb}$ and $\mathrm{S}$. Phase 2 has 4 times higher proportion of $\mathrm{Pb}$ than phase 1. Note overlap between $\mathrm{Pb} \mathrm{M}$ and $\mathrm{S} K$ peaks.

Fig.4 Examples of single pixel spectra for each phase simulated with approximately 150 counts in the energy region $2.2-2.6 \mathrm{keV}$. 